\title{
Alcune considerazioni sull'ipotesi di Sapir-Whorf applicata alla traduzione dei termini costituzionali italiani e spagnoli nell' ottica comparativo-contrastiva
}

\author{
Patrizia Brugnoli
}

The main aim of this article is to contribute to raising the interest around legal language, rarely analysed in the Italian-Spanish translation perspective. In particular, the author's interest is concentrated on specific normative texts, the Italian and Spanish Constitutions, of which she takes some lexical features into account. The article shows, through the use of componential analysis, that the "translatability" of the above mentioned traits of legal text from one language to another is impossible, even for those languages which are considered "similar" like Spanish and Italian. In Linguistics this hypothesis was supported by Sapir-Whorf and is particularly true if applied to the legal language. In fact, legal translation is "culture-bond" and it is intimately related to the socio-economic structures which are peculiar to a country.

\section{Introduzione}

La nostra esposizione si prefigge l'obiettivo di stimolare l'interesse e attirare l'attenzione intorno alla lingua speciale giuridica, scarsamente studiata nella prospettiva linguistica comparativo-contrastiva italiano-spagnolo.

Una prima osservazione su questa lingua speciale è che la lingua giuridica non è affatto una lingua universale: essa è parte integrante della tradizione e dell'unicità dell' ordinamento giuridico proprio di un paese, ed è legata intimamente al sostrato culturale di quel paese, cosicché persino i giurisperiti stessi incontrano difficoltà nel comprendere le peculiarità della lingua giuridica di un paese diverso dal proprio (Liaño 1996: 10; Hernandez Gil 1976:17). Tali difficoltà naturalmente aumentano se si passa da un sistema giuridico di base romanico-germanica, come quello italiano e spagnolo, a un sistema di Common Law, ovvero basata sul diritto giurisprudenziale e consuetudinario, come - ad esempio - quello inglese. Tuttavia, l'intento del presente articolo è proprio quello di riequilibrare il dibattito esistente in merito alle problematiche traduttive di un testo di diritto, praticamente monopolizzato dalle considerazioni sull' "impossibilità" o, comunque, sulla difficoltà di tradurre dei testi appartenenti uno al sistema di Civil Law e l'altro al sistema di Common Law. In realtà, i problemi traduttivi investono anche testi appartenenti al medesimo sistema giuridico, come ad esempio un testo normativo italiano e spagnolo.

Infatti, perché una lingua giuridica sia traducibile (qui "traducibile" deve essere inteso in senso ampio, ovvero sia comparabile) da una lingua naturale ad un' altra, deve esistere un "ragionevole grado di isomorfismo, sia 
a livello di strutture dell'espressione che a livello di strutture del contenuto nelle due lingue" (Cecioni 1996:157).

La "traducibilità" di un testo da una lingua naturale ad un'altra sarà, alla luce di quanto appena detto, tanto più difficile se è un testo giuridico a dover essere compreso e analizzato, poiché la lingua giuridica attiene a strutture socio-politiche talmente specifiche da non risultare mai completamente isomorfe, neanche tra paesi che apparentemente sono culturalmente affini, come l'Italia e la Spagna. Il problema della traducibilità di un testo giuridico è molto più complesso di quanto possa sembrare, proprio perché investe il rapporto tra lingua e cultura. I problemi pertanto che si potranno riscontrare in un testo giuridico non saranno solamente di interferenze linguistiche, ma - e soprattutto - quello relativo alle interferenze giuridiche. Nel primo caso la metodologia rigorosa che si dovrà adottare nella traduzione sarà la linguistica comparata, mentre nel secondo caso sarà il diritto comparato.

In questa sede viene osservato in particolare il livello linguistico che forse più caratterizza e contribuisce a rendere il testo normativo, e in particolare quello costituzionale, peculiare: il livello lessicalo-semantico.

\section{L'ipotesi di Sapir-Whorf e l'intraducibilità dei termini costituzionali italiani e spagnoli}

La traduzione è un'operazione duplice: da un lato prende in considerazione la distanza culturale e, dall'altro, quella linguistica tra il testo di partenza e la lingua/cultura del testo di arrivo. La conseguenza pratica di ciò è che il traduttore deve avere sia competenze linguistiche, sia culturali. Ma che tipo di relazione esiste tra lingua e cultura? Secondo Whorf, la lingua è un sistema per plasmare la conoscenza, uno strumento cognitivo: più concretamente la lingua serve per ordinare la conoscenza; perciò se due discenti parlano due lingue diverse non usano solo due formulazioni diverse dello stesso concetto, bensì hanno anche due visioni del mondo diverse (Whorf 1956: 15). Imparare un'altra lingua significa, pertanto, acquisire la visione del mondo di quella lingua. Per questo tra discenti che parlano lingue differenti non vi può essere una conoscenza univoca e una stessa visione del mondo. Che conseguenze ha sulla traduzione questa teoria? La specificità culturale e linguistica di cui si è parlato finora risulterebbe nell'impossibilità di comunicare e di tradurre. E' questa la teoria della relatività linguistica, la cosiddetta "ipotesi Sapir-Whorf", secondo la quale, "la struttura della lingua condizionerebbe la struttura stessa del pensiero dei parlanti quella lingua (1956: 15). Corollario sarebbe allora che, siccome le lingue sono diverse, anche $i$ concetti sono diversi da cultura a cultura e da società a società" (Berruto 1979:56).

Secondo questa visione, non si può, pertanto, considerare universale il significato di una parola in varie culture e società, poiché il significato è relativo solo a un certo contesto socioculturale. Ne consegue che la lingua non può essere vista come una nomenclatura, ossia è impensabile che i significati delle parole ricevano etichette differenti nelle diverse lingue. Sapir, 
infatti, afferma: "non esistono due lingue che siano sufficientemente simili da essere considerate come rappresentanti della stessa realtà sociale. I mondi in cui vivono differenti società sono mondi distinti, non sono semplicemente lo stesso mondo con etichette differenti" (1972:58).

Questo è anche il concetto ribadito da Viezzi (1994:15) che, a proposito della traduzione del lessico giuridico, nega che possa esistere un sistema di nomenclature parallele per un referente universale $x$, mentre ribadisce l'esistenza di un referente $x, y$ definiti solo in quanto disciplinati da un dato sistema giuridico. Dal punto di vista pratico, questa teoria porterebbe, quindi, all'intraducibilità dei termini giuridici, anche tra lingue e culture come quella italiana e spagnola.

Per capire a fondo la questione della traducibilità o, forse, dovremmo dire dell'intraducibilità di fondo dei termini costituzionali, proviamo a fare un'operazione di analisi di diritto comparato tra alcuni termini italiani e spagnoli che designano le fonti del diritto dei due paesi. A tale fine, abbiamo portato degli esempi d'analisi contrastiva e componenziale dei termini giuridici italiani e spagnoli che, almeno a uno sguardo sommario, potrebbero sembrare pressoché uguali in quanto aventi lo stesso referente nei due diversi ordinamenti. Newmark (1988:114) afferma che se l'analisi componenziale applicata alla linguistica individua i tratti semantici o semi di una parola, quella applicata alla traduzione è fondamentalmente un' analisi comparativa e contrastiva tra due termini di due lingue, termini simili nel significato, ma che non sono perfettamente equivalenti. L'operazione comparativa e contrastiva tra $\mathrm{i}$ termini costituzionali presi in considerazione ha applicato proprio l'analisi componenziale usata nella traduzione, analisi che ci permette di scomporre e ricomporre i semi di un termine per confrontarli con i semi contenuti nel termine corrispondente della seconda lingua. Anche se Newmark ribadisce che questo metodo è utilizzato principalmente "in translating cultural (and institutional) words that the readership is unlikely to understand" (1988:119), tuttavia esso si rivela fruttuoso anche per studiare quei termini appartenenti a lingue e culture affini.

\section{L'analisi componenziale applicata ai termini giuridici italiani e spa- gnoli: quattro esempi pratici}

Partiamo dal primo esempio: è corretto tradurre "leyes de reforma de la Constitución" con "legge di revisione costituzionale"? Si corrispondono i due tipi di fonti? Fino a che punto l'uno traduce l'altro? Il referente può dirsi lo stesso? Secondo la definizione del monolingue giuridico spagnolo, le "leyes de reforma de la Constitución" sono quelle che "añaden, suprimen o sustituyen algún precepto de la Constitución. " (DSPE 1984:344). Un raffronto linguistico contrastivo col sistema italiano ci porta a concludere che le "leyes de reforma de la Constitución" non includono solamente le "leggi di revisione costituzionale", bensì anche le "leggi costituzionali". Infatti, se la definizione di "leyes de reforma de la Constitución " include anche l'idea di integrare (añadir) la Costituzione spagnola, allora tale definizione cor- 
risponde alla definizione italiana di "legge costituzionale": infatti, le "leggi costituzionali" italiane per antonomasia non sono chiamate ad alcuna sostituzione del testo costituzionale. Questo vuol dire, in pratica, che la locuzione spagnola "leyes de reforma de la Constitución" congloba dal punto di vista referenziale sia quella di "legge costituzionale", sia quella di "legge di revisione costituzionale". In realtà, però, si è già detto che il sintagma "leggi costituzionali" include sia quelle leggi che attuano disposizioni della Costituzione (sono queste le vere e proprie "leggi costituzionali"), sia le leggi che abrogano o modificano parte della Costituzione (sono queste le "leggi di revisione costituzionale"). Come ha ben sottolineato Falcon, "legge costituzionale e legge di revisione costituzionale non sono ... fonti diverse, ma nomi diversi attribuiti alla stessa fonte, a seconda che si tratti di modificare o solo attuare il testo costituzionale" (1991:217).

Passiamo ora ad un secondo esempio: come si traduce la locuzione "revisione costituzionale" in spagnolo? Corrisponde alla "reforma constitucional" o alla "revisión constitucional", o ad entrambe? Per quanto concerne la procedura di revisione costituzionale, la Costituzione italiana dedica solamente l'art. 138, mentre la Costituzione spagnola utilizza un intero Titolo (il Titolo X, in particolare gli art. $166 \mathrm{sgg}$.) per le procedure di revisione costituzionale. Aparicio (1994:184) fa notare che la Costituzione spagnola usa due termini per indicare quello che la Costituzione italiana chiama semplicemente "revisione costituzionale". Infatti, nel Titolo citato, la Costituzione spagnola parla di "reforma" (Cost.sp., art. 167) e di "revisión" (Cost. sp., art. 168), distinzioni terminologiche che non sono contemplate nella Costituzione italiana. La Costituzione spagnola, infatti, parla di "reforma" quando indica "la modificación parcial de las que podríamos denominar partes ordinarias o simples de la propia Constitución, independientemente del número de preceptos cuya modificación se proponga" (cors. agg.) (Aparicio 1994:184). Col termine "revisión", invece, la Costituzione spagnola indica "la modificación de la totalidad de la Constitución o de partes que se consideran fundamentales o sustanciales ..." (cors. agg.) (1994:184). Da ciò ne discende che la traduzione è impossibile poiché l'oggetto giuridico-referente, al quale si ricollega la locuzione "revisione costituzionale", non trova un corrispondente esatto nell'oggetto giuridico-referente della locuzione "revisión constitucional" dell'ordinamento spagnolo.

Un terzo esempio: si può infine ravvisare un'equivalenza terminologica e giuridica perfetta tra le "leyes orgánicas" spagnole e le "leggi costituzionali" dell'ordinamento italiano? V'è lo stesso referente per i due termini? Seguendo i manuali di diritto costituzionale italiano e spagnolo, le materie di competenza delle une e delle altre sono differenti e, così, le materie oggetto di "reserva orgánica" e di "riserva costituzionale". Inoltre le "leggi costituzionali" sono pariordinate rispetto alla Costituzione, mentre le "leyes orgánicas" si trovano sottordinate rispetto alla Carta costituzionale spagnola. Un'ulteriore differenza tra le "leyes orgánicas" e le "leggi costituzionali" sta nel fatto che mentre le "leggi costituzionali" sono approvate con la stessa procedura aggravata prevista per la revisione della Costituzione italiana all'art. 138, le "leyes orgánicas" invece sono approvate con proce- 
dura prevista dallo stesso art. 81 che le disciplina . Tale articolo prevede una procedura e maggioranze diverse da quelle previste per la "reforma" e la "revisión", rispettivamente regolate dagli artt. 167 e 168 della Costituzione spagnola. Un'altra osservazione da ricollegare all' approvazione delle "leyes orgánicas" è che la Costituzione spagnola, ex art. 81, richiedendo la maggioranza assoluta per la sola Camera dei Deputati, configura una situazione di disparità tra le due Camere, situazione che non si prospetta in Italia poiché nella procedura di revisione della Costituzione le maggioranze richieste sono le stesse per entrambe le Camere. Infatti, in Spagna, esiste il cosiddetto "bicameralismo imperfetto", mentre in Italia vige il "bicameralismo perfetto".

Ed infine, un quarto esempio: la locuzione "pubblico ministero" traduce lo spagnolo "ministerio fiscal"? L'articolo 124, 1 della Costituzione spagnola fornisce una definizione di "ministerio fiscal", ossia di quell'organo che:

Tiene por misión promover la acción de la justicia en defensa de la legalidad, de los derechos de los ciudadanos y del interés público tutelado por la ley, de oficio o a petición de los interesados, así como velar por la independencia de los Tribunales y procurar ante éstos la satisfacción del interés social.

La Costituzione italiana all'art. 107 u.c. prevede la figura del Pubblico ministero che viene definito attraverso i suoi compiti:

veglia sull'osservanza delle leggi, alla pronta e regolare amministrazione della giustizia, alla tutela dei diritti dello Stato, delle persone giuridiche e degli incapaci, richiedendo nei casi d'urgenza i provvedimenti che ritiene necessari; promuove la repressione di reati e l'applicazione delle misure di sicurezza: fa eseguire i giudicati ed ogni altro provvedimento del giudice, nei casi stabiliti dalla legge (NDG 1998:973, voce "Pubblico ministero").

Di conseguenza "ministerio fiscal" ha un referente diverso rispetto a "Pubblico ministero", anche perché nella pratica il "ministerio fiscal" spagnolo può avere come equivalente anche una serie di denominazioni che il Pubblico ministero italiano assume a seconda del giudice presso il quale esercita le sue funzioni, denominazioni che rispecchiano i diversi livelli organizzativi di questo ufficio: il Procuratore generale presso la Corte di Cassazione, le Procure generali presso le Corti d'appello, le Procure della Repubblica presso i tribunali ordinari, i tribunali per i minorenni.

\section{Conclusioni}

Quanto dimostrato nell'articolo ci porta a concludere che se l'ipotesi di Sapir-Whorf fosse applicata alla lettera, i testi (e in modo particolare quelli giuridici) diverrebbero intraducibili, poiché mancherebbero le premesse per una comunicazione tra membri di comunità linguistiche diverse e, ancor di più, tra comunità giuridiche diverse. La diversa percezione e organizzazione mentale della realtà può essere usata per spiegare l'esistenza di certi "vuoti" 
tra lingue e tra culture (anche giuridiche), vuoti che possono rendere la traduzione un compito alquanto difficile. Con riferimento alla traduzione giuridica, il pericolo insito è che, nell'affanno di attuare un'operazione di mediazione culturale tra ordinamenti e lingue "simili" come quelli italiani e spagnoli, molti di questi "vuoti" giuridici tra i due paesi vengano appiattiti, dando luogo a errori grossolani di traduzione. Come abbiamo ampiamente dimostrato, pur non esistendo nelle due culture giuridiche trattate veri e propri "vuoti referenziali", non sono rinvenibili delle categorie di referenti giuridici uguali e totalmente sovrapponibili. L'intraducibilità allora deve essere vista come una non totale omologazione referenziale, ovvero un valore positivo di sensibilità interculturale.

\section{Bibliografia}

Ambruzzi, Luciano (1973). Nuovo dizionario spagnolo-italiano, italiano-spagnolo. Torino: Paravia.

Aparicio, Miguel Angel (1994). Introducción al sistema político y constitucional español. Barcelona: Ariel.

Berruto, Gianni (1979). La Semantica. Bologna: Zanichelli.

Brugnoli, Patrizia (1999-2000). Analisi interlinguistica ed interdisciplinare delle Carte costituzionali italiana e spagnola. Tesi di dottorato di ricerca non pubblicata, Università di Trieste.

Brugnoli, Patrizia (2002). "Alcune riflessioni sulle problematiche dei termini politico-istituzionali nella Costituzione italiana e spagnola." Translation Journal 6. in rete, URL: http://accurapid.com/journal/20legal.htm (consultato 27.09.02).

Brugnoli, Patrizia (2002). "Il piano lessico-semantico della lingua costituzionale italiana e spagnola come strumento di democrazia." Terminologie et Traduction. European Commission: Luxembourg (3), 160-171.

Brugnoli, Patrizia (in corso di stampa). "La lingua giuridica va riformata? Alcune riflessioni sul dibattito in corso tra giuristi e linguisti." Revista de Llengua $i$ Dret.

Carroll John (1956, 1991). Selected Writings of Benjamin Lee Whorf, Language, Thought and Reality. Cambridge/Massachusetts: the MIT Press.

Cecioni, Giuseppina (1996). "La Traducibilità del linguaggio giuridico inglese." G. Cortese (a cura di) (1996). Tradurre i linguaggi settoriali. Atti del seminario di Anglistica nell'ambito del progetto strategico CNR "I problemi della traduzione nell'Italia dell'Europa". Università degli studi di Torino. Facoltà di Scienze Politiche 27-28 maggio 1993. Torino: Edizioni Libreria Cortina, 155-173.

Constitución española (1993). Biblioteca de Legislación Serie Menor. Madrid: Gredos.

Costituzione della Repubblica italiana (1995). Padova: Cedam.

DC $(1995)=$ Diritto costituzionale. Napoli: Edizioni Simone.

DRAE (1998) = Diccionario de la lengua española . Madrid: Espasa-Calpe.

DSPE (1984) = González Encinar, J. J. (ed.) (1984). Diccionario del sistema político español. Madrid: Akal.

Falcon, Gianni (1991). Lineamenti di diritto pubblico. Padova: Cedam.

Hernández Gil, Armando (1976). Estructuralismo y derecho. Colección Alianza Universitaria. Madrid: Alianza. 
Liaño, Manuel (1996). "Las peculiaridades del lenguaje jurídico desde la perspectiva del jurista.” P. San Ginés Aguilar \& E. Ortega Arjonilla (editori) (1996). Introducción a la Traducción jurídica y jurada (inglés-español). Granada: Editorial Comares, 9-24.

NDG (1998) = Del Giudice, F. (ed.) (1998). Nuovo Dizionario Giuridico. Napoli: Edizioni Simone.

Newmark, Peter (1988). A Textbook of Translation. Hemel Hempstead/Hertfordshire: Prentice Hall International.

Pérez Royo, Juan (1998). Curso de Derecho Constitucional. Madrid: Ediciones Marcial Pons.

Sapir, Edward (1972). Cultura, linguaggio, personalità. Torino: Einaudi.

Viezzi, Maurizio (1994). "Introduzione alle problematiche della traduzione giuridica con particolare riferimento alla traduzione di testi in lingua inglese." Giuseppe Di Mauro \& Federica Scarpa (editori) (1994). Traduzione, Cultura e Società 5. Trieste: LINT, 3-48.

Whorf, B.L. (1956). "The Relation of Habitual Thought and Behavior to Language." J. B. Carroll (ed.) (1956). Language, Thought and Reality. Selected Writings of Benjamin Lee Whorf. Cambridge/Massachusetts: the MIT Press, 134-159.

Zingarelli (1988) = Dogliotti, Maurizio \& Luca Rosiello (editori) (1988). Il nиоvo Zingarelli. Vocabolario della lingua italiana. Bologna: Zanichelli. 